\section{Ambulatory Oncology Operations: Strategies to Alleviate Complexity}

\author{
Chadi Nabhan, MD, MBA, and Sandeep Parsad, PharmD
}

Although 1.8 million new cancer cases are detected annually, most patients who
are diagnosed remain alive at 5 years. ${ }^{1}$ Factors contributing to this success include
advancements in science, better supportive care, and technological progress. ${ }^{2}$
Addressing the needs of patients with cancer has become a major operational
challenge as more cancers are detected earlier and more patients are living longer.
Patients receive complex, multimodality therapy in a variety of settings, including
the integration of various disciplines. Coordination of care for newly diagnosed
patients-often an urgent scenario requiring rapid diagnostic studies and treatment
initiation-is essential for success.

Care for survivors also requires coordination, with similar implications on cancer care operations. Although the increase in the number of patients with cancer and the associated revenues from these cases have led academic and community institutions to declare cancer as the premier service line, delivering cancer care efficiently and effectively remains an operational contest with no easy answers. This challenge is increasingly obvious as cancer care delivery is shifted to the outpatient arena and as the number of oncologists in the United States is projected to fall short of increased demand. ${ }^{3}$

\section{Factors Affecting Outpatient Delivery of Cancer Care}

In addition to a growing population of patients with cancer as a result of advances in earlier detection and the success of prolonged survival, the economy, new regulations, and technology are factors that impact ambulatory oncology operations.

\section{Economy}

Review of the overall fiscal environment suggests a healthy US economy with an expected yearly gross domestic product (GDP) growth of $2 \%$ to $3 \%$ for the next 3 years. Unemployment is expected to be between $4 \%$ to $5 \%$ during the same time period. ${ }^{4}$ In addition, despite a minimal increase in wages, current projections suggest that the stock market could reach new highs and the housing market will strengthen in the future.

Collectively, these factors imply economic prosperity and better cash reserves for consumers, which potentially translates into better access to clinics and physicians. As more patients enter the healthcare market, cancer diagnoses could further increase as a natural result of increased screening. Deductibles, copays, and out-of-pocket expenses that are known to be barriers to medical care become less daunting with higher disposable income. Thus, an economic upturn calls for a strategic assessment of how to improve access to cancer clinics across the country efficiently and effectively.

\section{Government Regulations and the Affordable Care Act}

For the near future, the Affordable Care Act (ACA) will influence healthcare delivery, ${ }^{5}$ and a few key items in the ACA pertain to ambulatory oncology operations. First, as more patients enter the medical system due to insurance availability, more attention will need to be focused on access and ease of appointments. Institutions already overwhelmed with the current volume of patients will need to prepare for a further influx. This is of paramount importance in the cancer service line, as patients demand timely visits after a cancer diagnosis. Second, the assumed lack of familiarity with the ACA and

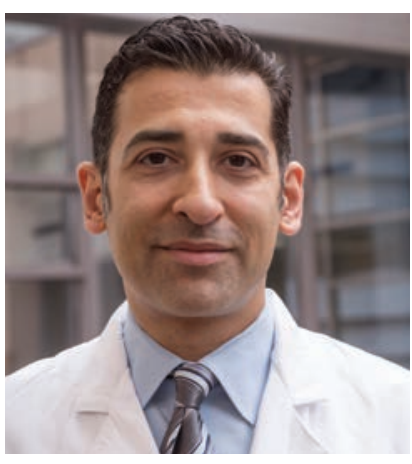

Chadi Nabhan, MD, MBA

Chadi Nabhan, MD, MBA, is a hematologist and medical oncologist with research focus on the diagnosis and management of hematologic malignancies (leukemias and lymphomas), as well as prostate cancer.

Dr. Nabhan is Vice President and Chief Medical Officer at Cardinal Health Specialty Solutions. Prior to joining Cardinal Health, Dr. Nabhan oversaw clinical cancer services and operations at the University of Chicago Medicine, where he supervised medical, surgical, and radiation oncology. Dr. Nabhan has been the primary investigator for many clinical trials, and some of his research work presented at national and international meetings. $\mathrm{He}$ is interested in investigating racial, ethnic, gender and age disparities in treatment approaches and outcomes for patients with lymphoid malignancies and CLL. Dr. Nabhan is interested in developing strategies to improve cancer care delivery in the era of value-based care and has a focus on the business and economic aspect of oncology as they impact all stakeholders involved. Dr. Nabhan has more than 200 peer-reviewed articles, abstracts or book chapters and serves on editorial boards of multiple scientific journals, including JAMA-Oncology. He is also a reviewer for major medical journals, including JAMA, JAMA-Oncology, Blood, Annals of Oncology, European Journal of Hematology, and British Journal of Hematology. 


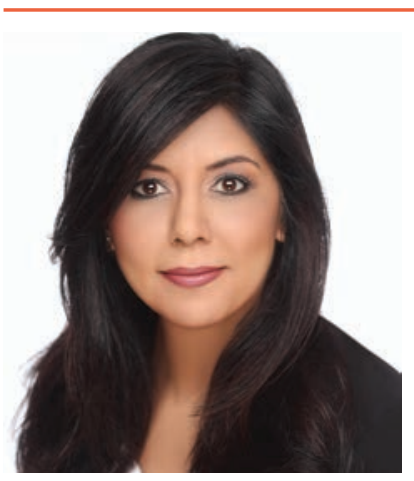

Sandeep Parsad, PharmD

Sandeep Parsad, PharmD, is a clinical oncology pharmacist at The University of Chicago Medicine. She is Director of the PGY2 Oncology Pharmacy Residency Program, CoChair of the Oncology Drug Safety Committee and directs Investigational Drug and Hematology/Oncology pharmacy services. Dr. Parsad's interests include stem cell transplantation, hematologic malignancies, value-based payment models, and oncology quality initiatives. new healthcare coverage requires ambulatory oncology centers to invest in financial planners or counselors to help patients navigate this complex healthcare process.

Further, the focus on quality metrics and "pay-for-performance" directly affects ambulatory oncology operations. ${ }^{6}$ Visits in ambulatory cancer clinics may require a longer time to account for these new measures. The emphasis of the ACA on coordinating seamless care for patients underscores the need for ambulatory operations to focus on access, synchronization, and patient satisfaction. In most settings, hospitalized patients with cancer will need to be seen in ambulatory clinics within 48 hours of discharge. This goal may be unattainable without prospective planning. Collectively, measures embraced by the ACA will require meticulous attention by ambulatory cancer care centers to maintain revenues. Notably, the Centers for Medicare \& Medicaid Services has embarked on several innovation grants that foster ACA principles (https:// innovation.cms.gov). The core idea of these grants is for cancer institutions to become innovative in coordinating care. In theory, this innovation could to lead to improved quality and outcomes, lowered or maintained costs, and restored patient satisfaction.

\section{Technology and Trends}

Patients are increasingly using new technology to access healthcare information, reach physicians, and schedule appointments and follow-ups. The coordination of technology and how it is used and delivered to the patient presents another opportunity for improving ambulatory oncology operations. Enhancing patientphysician communication platforms through patient portals as part of electronic medical records is already underway. Innovative and forward-thinking institutions have already started investing in mobile apps to facilitate online scheduling, arguing that these strategies facilitate access. These initiatives must be properly coordinated, user-friendly, and reliably available across disciplines.

A few market "disrupters" may also impact cancer operations. "Health wearables" such as fitness watches and the desire of companies such as Google and Apple to enter the healthcare market with similar products can affect patients with cancer, who may be overwhelmed with information from various sources. Direct-to-consumer advertising about genetic testing, screening, and some treatments could further overwhelm ambulatory oncology operations.

Moreover, patients with cancer are increasingly interested in complementary medicine, such as massage therapy, herbal therapy, nutrition, and acupuncture, and how they may be integrated within cancer care. Oncologists need to recognize these trends and how they might affect their interactions with patients.

Several trends in oncology practices across the country are also noteworthy. Economic pressures and reimbursement changes are causing many practices to consolidate or join larger institutions and hospitals. These mergers and acquisitions are likely to continue as integration networks ultimately grow. This trend directly affects ambulatory oncology operations because assuring the same quality of care delivery across sites belonging to the main entity is essential. The complexity of this process cannot be underestimated and requires strategic thinking on proper implementation.

\section{Proposed Solutions}

Although a universal solution to the complexity of ambulatory oncology operations is not practical, several proposed strategies can improve the process. These strategies include:

- Better utilization of advanced practice providers by allowing them to see a larger number of return patients; this can provide new patients with faster access to medical oncologists; 
- Creating consistent available visit times for discharged patients and urgent visits;

- Investigating a nurse navigation model in which nurses are linked to a disease type and help provide patients with a central point of contact through their entire cancer journey ${ }^{7}$; importantly, nurse navigators need to focus on education and clinical care as opposed to nonclinical administrative tasks;

- Exploring supportive oncology programs to provide complementary medicine under the supervision of well-trained personnel; as this is a clear trend, assuring that proper information is being transmitted to patients is essential;

- Creating a pharmacist-led clinic to assure adherence to oral chemotherapy drugs, provide education, assess for toxicity, and effectively monitor for drug-drug or drug-food interactions; and

- Investing in survivorship programs in which patients can receive follow-up care after active anticancer therapy has completed.

\section{Conclusions}

The shortage of oncologists in the United States, ${ }^{3,8}$ coupled with increased numbers of patients with cancer being treated and changes in the economy, technology, and regulations, exert enormous pressure on ambulatory oncology centers. More attention to proper strategies to enhance these operations in the years ahead is urgently needed.

\section{References}

1. Siegel RL, Miller KD, Jemal A. Cancer statistics, 2016. CA Cancer J Clin 2016;66:7-30.

2. Dizon DS, Krilov L, Cohen E, et al. Clinical cancer advances 2016: annual report on progress against cancer from the American Society of Clinical Oncology. J Clin Oncol 2016;34:987-1011.

3. Yang W, Williams JH, Hogan PF, et al. Projected supply of and demand for oncologists and radiation oncologists through 2025: an aging, better-insured population will result in shortage. J Oncol Practice 2014;10:39-45.

4. U.S. Economy at a Glance: Perspective from the BEA accounts. Bureau of Economic Analysis Web site. Available at: http://www.bea.gov/newsreleases/glance.htm. Accessed September 26, 2016.

5. McDonough JE. The road ahead for the Affordable Care Act. N Engl J Med 2012;367:199-201.

6. Jha AK, Joynt KE, Orav EJ, Epstein AM. The long-term effect of premier pay for performance on patient outcomes. N Engl J Med 2012;366:1606-1615.

7. McMullen L. Oncology nurse navigators and the continuum of cancer care. Semin Oncol Nurs 2013;29:105117.

8. Hortobagyi GN. A shortage of oncologists? The American Society of Clinical Oncology workforce study. J Clin Oncol 2007;25:1468-1469. 